\title{
Effect of Grain Boundaries on the Vibrational Properties of Phononic Crystals Ralf Meyer $^{1}$ \\ ${ }^{1}$ Department of Mathematics and Computer Science and Department of Physics, Laurentian University, 935 Ramsey Lake Road, Sudbury, ON P3E 2C6, Canada
}

\begin{abstract}
The influence of grain boundaries on the vibrational properties of nanoscale phononic crystals is studied with the help of molecular dynamics simulations. The low-frequency vibrational density of states of phononic crystals made from single crystal and polycrystalline silicon are derived from the simulations. The results show that the presence of grain boundaries leads to an increase of the density of states and a change of its peak structure at low frequencies. Calculations of the band structure of the model systems along one direction reveal that the grain boundaries affect the bands differently and in a non-uniform manner.
\end{abstract}

\section{INTRODUCTION}

Phononic crystals are artificially created materials that promise to be useful in numerous applications. Phononic crystals are characterized by periodic variations of their elastic properties, which make it possible to control the propagation of elastic waves in the material. Phononic crystals can be designed to have vibrational band gaps, i.e. forbidden frequency ranges in which vibrational waves cannot travel through the material. An overview of the physics of phononic crystals can be found in references [1-3].

It has been shown theoretically [4] and experimentally [5,6] that phononic crystals with periodicity length on the nanometer scale can have reduced thermal conductivities. This makes them candidates for thermoelectric materials with high figures of merit $Z T$ and has raised interest in the properties of thermal phonons in phononic crystals. In this study, the vibrational properties of nanoscale silicon phononic crystals are calculated from molecular dynamics simulations. The effect of grain boundaries on the vibrational spectrum is studied by comparing the properties of phononic crystals with and without grain boundaries. Results are shown for the low frequency vibrational density of states as well as the band structure along one direction in $k$-space.

\section{COMPUTATIONAL DETAILS}

Two similar model configurations have been used for the molecular dynamics simulations in this work. Both configurations consist of 72 silicon nanoparticles with a diameter of $9 \mathrm{~nm}$ that are placed on the sites of a honeycomb lattice with a nearest-neighbor distance of $16.7 \mathrm{~nm}$. The nanoparticles are connected through silicon nanowires with a diameter of $6 \mathrm{~nm}$. One of the two model configurations was cut out of a block of single crystal silicon and contains no grain boundaries. The other configuration was built from nanoparticles and nanowires with different crystalline orientations. This configuration is polycrystalline with grain boundaries at the junctions between particles and wires. Figure 1 shows the polycrystalline configuration as an example for the model systems simulated in this work. 


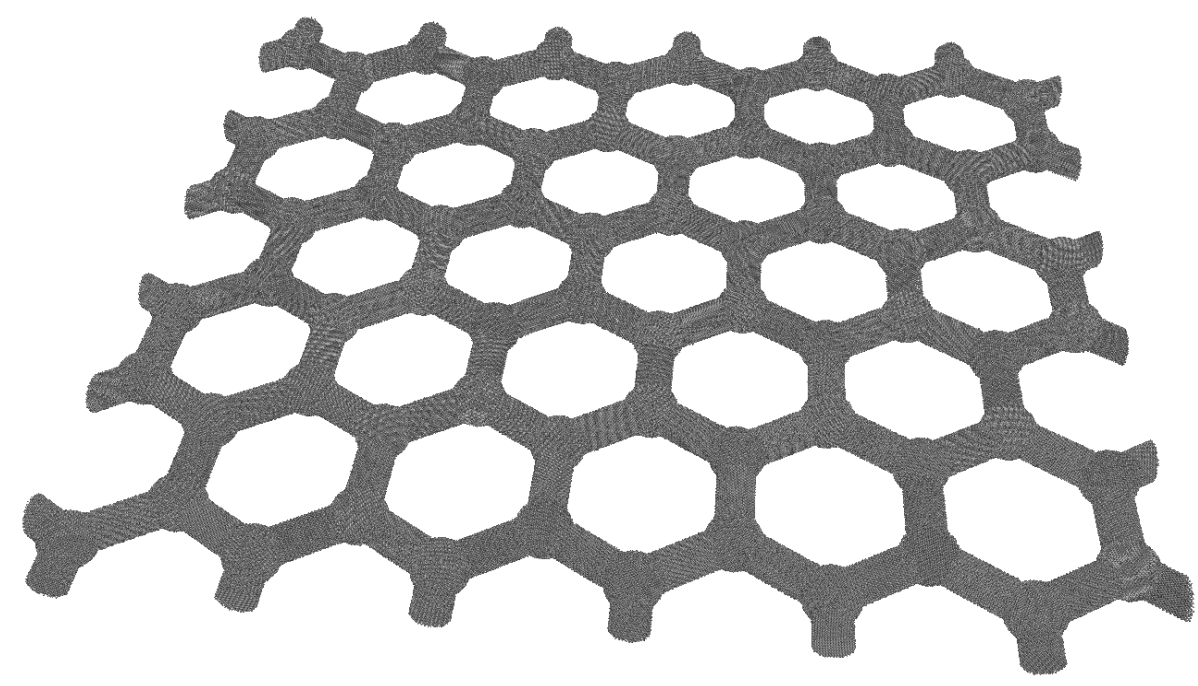

Figure 1. Polycrystalline phononic crystal model built from silicon nanowires and nanoparticles.

The phononic crystal models simulated in this work are similar to the model used in reference [7]. With a size of 2.7 million atoms the models here are, however, smaller than the 9 million atom model used in that article. Also, the model systems in this work contain nanoparticles with the same diameter whereas the model in the earlier work consists of nanoparticles with two different sizes on the sites of a binary honeycomb lattice.

The molecular dynamics simulations of the two model configurations employed an MEAM potential [8] for the calculation of the inter-atomic forces. The simulations were carried out at a temperature of $300 \mathrm{~K}$ using the velocity Verlet algorithm [9] with a time step of $1 \mathrm{fs}$. Periodic boundary conditions were applied along the $\mathrm{x}$ and $\mathrm{y}$ directions. After a careful equilibration, both configurations were simulated over a period of nearly 2 million simulation steps.

The vibrational properties of the simulated systems were derived from the simulations with the help of the velocity autocorrelation function. The total vibrational densities of states of the model systems were obtained by Fourier transformation of the velocity autocorrelation function. The frequencies of individual modes were derived from the Fourier spectrum of the autocorrelation functions of velocities projected onto plane waves [10].

For large systems, the size of the data volume makes it impracticable to use the velocity data of all atoms for the calculation of velocity autocorrelation functions. To overcome this problem, a previously introduced sampling method was employed which calculates the velocity autocorrelation functions from the velocity data of a subset of the atoms [7]. From each of the configurations, 250,000 atoms were randomly selected and the velocities of these atoms were stored during the simulations every 15 simulations steps.

\section{RESULTS AND DISCUSSION}

Figure 2 shows the vibrational density of states at low frequencies obtained from molecular dynamics simulations of the two model phononic crystals. The densities of states shown in this figure have been slightly broadened by a finite line width of $1 \mathrm{GHz}$ in order to reduce the noise. Due to the finite length of the simulations, noise is a typical problem for calculations of 


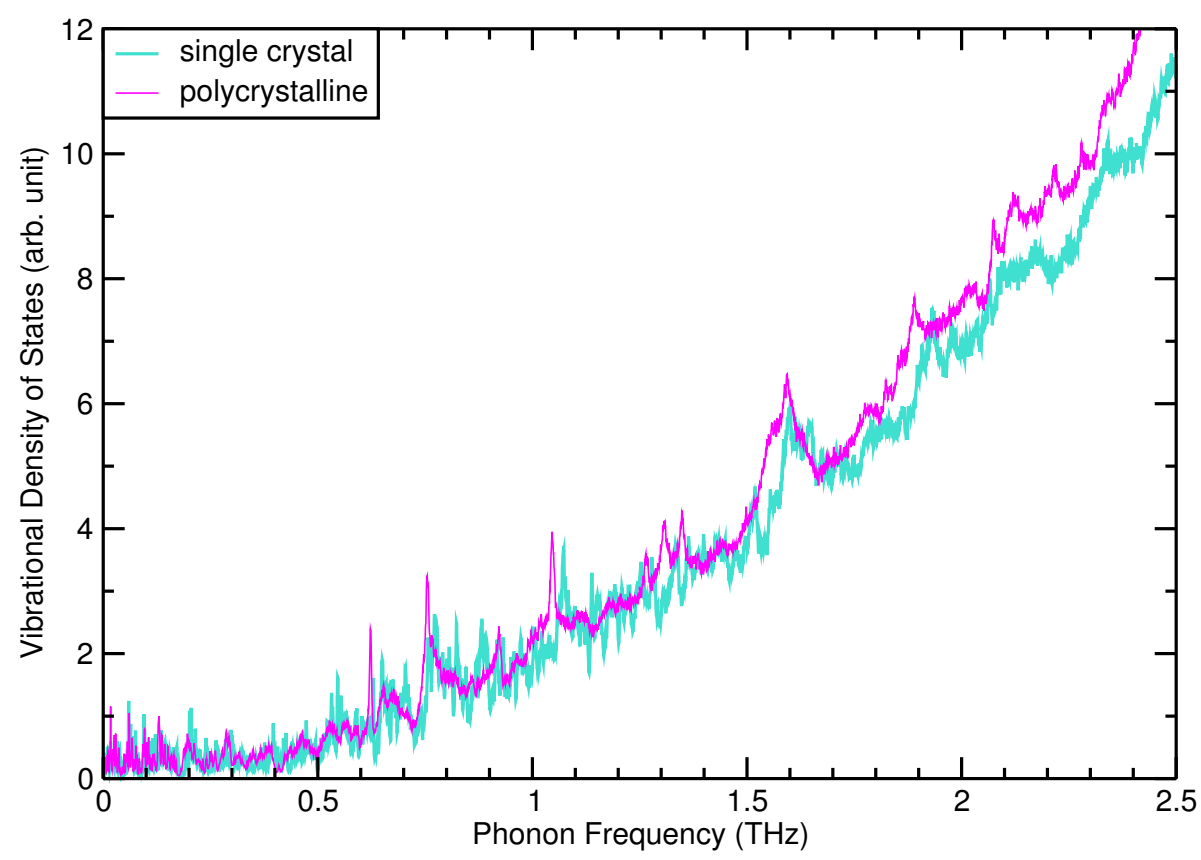

Figure 2. Low-frequency vibrational density of states of single crystal and polycrystalline silicon phononic crystal models calculated from molecular dynamics simulations.

vibrational densities of states from molecular dynamics simulations. In many cases this problem can be solved through a broadening of the data with a finite line width. In this work, however, this is difficult since we are interested in the low-frequency part of the density of states. This prohibits the use of a large line width since the line width sets a natural limit to the lowest frequencies that can be analyzed. The value of $1 \mathrm{GHz}$ used in this work is therefore a compromise, which provides only a partial solution to the noise problem.

Another problem for the determination of the low-frequency vibrational density of states is the finite size of the system. When the wavelengths of the modes become comparable to the system size, only a small number of different modes is compatible with the boundary conditions of the system. This means that the discrete nature of the spectrum of a finite system becomes visible and the concept of a density of states no longer applies. For the model systems in this work, this effect should only play a role for frequencies below $200 \mathrm{GHz}$. The vibrational properties of the phononic crystal models for these very low frequencies will be discussed below using the band structure. Most of the frequency range of figure 2 should not be affected by the finite size of the simulated systems.

Figure 2 shows two main differences between the two phononic crystal models. Firstly, the low-frequency vibrational density of states of the polycrystalline model is increased compared to the single crystal model. Secondly, the peak-structures of the density of states differ notably in the range from 500 to $1200 \mathrm{GHz}$.

The increase of the low-frequency vibrational density of states in the polycrystalline model system compared to the single crystal model indicates that the presence of grain boundaries leads to a decrease of the elastic constants. This behavior is expected since the grain boundaries should soften the system.

From figure 2 it can be seen that the low-frequency part of the vibrational density of states of both models has a complex structure with a number of sharp peaks. Although some of these 
peaks might be artifacts from noise, the larger peaks are clearly real features of the phononic crystals' density of states. As discussed below, the peaks at very low frequencies may be caused by the finite size of the simulated systems. For frequencies above $200 \mathrm{GHz}$, the peaks can be caused by either flat bands or van Hove Singularities, which occur when the gradient of a band vanishes at some point in the Brillouin zone.

A comparison of the density of states of the two model systems shown in figure 2 reveals that the polycrystalline model features fewer peaks than the single crystal model. This difference is unsurprising since the polycrystalline model is not perfectly periodic and the grain boundaries cause local disorder. Such disorders can broaden the peaks and remove sharp singularities. There are, however, some remarkable exceptions to this behavior, in particular the two peaks at 625 and $750 \mathrm{GHz}$. These peaks appear to be even higher in the polycrystalline model than in the single crystal model. The nature of these peaks and the reason why they remain so prominent in the polycrystalline model is yet unclear. A possible explanation is that these peaks are caused by modes that are localized inside either the nanoparticles or the nanowires. If this is the case, the presence of grain boundaries at the particle-wire junctions might increase the localization of these modes thereby stabilizing the peaks instead of destroying them. This hypothesis could be tested by separate calculations of the local vibrational of states for atoms in the nanoparticles and in the nanowires.

The vibrational band structure provides a deeper insight into the vibrational properties of a system than the density of states. Figure 3 shows the nine lowest bands of the calculated band structure of the two phononic crystal models along the $\Gamma-\mathrm{M}$ direction. As can be seen from the figure, the five lowest bands are very similar in both models, except for a slight shift of the bands to lower frequencies in the polycrystalline model. This shift is consistent with the increase of the density of states of the polycrystalline model shown in figure 2 .

For the remaining four bands, the differences between the two models are less uniform. While two of these bands are also shifted two lower frequencies, the other two are not. The band running from $177 \mathrm{GHz}$ at the $\Gamma$-point to $192 \mathrm{GHz}$ at the $\mathrm{M}$-point in the single crystal model is shifted upwards and runs from $181 \mathrm{GHz}$ to $197 \mathrm{GHz}$ in the model made from polycrystalline silicon. And while the highest band shown in figure 3 is shifted down by less than a $\mathrm{GHz}$ at the $\Gamma$-point in the polycrystalline model, it is shifted upwards by $10 \mathrm{GHz}$ at the $\mathrm{M}$-point. Since this band is very flat, the shift in opposite directions changes the sign of the slope of this band which implies a reversal of the group velocity.

It should be noted that even among the bands that are shifted to lower frequencies, the size of the shift is neither uniform along the bands nor between them. These differences lead to an increase of the band gap along the $\Gamma-\mathrm{M}$ direction between the fifth and sixth band. This gap increases from $12.2 \mathrm{GHz}$ in the single crystal model to $17.3 \mathrm{GHz}$ in the polycrystalline model.

\section{CONCLUSIONS}

This work compares the vibrational properties of two models for nanoscale phononic crystals built from nanowires and nanoparticles. While one of the models is made from single crystal silicon, the other is made from polycrystalline silicon with grain boundaries at the particle-wire junctions. The low-frequency vibrational density of states and band structure have been derived for both models from molecular dynamics simulations. Comparison of the results for both models sheds light onto the impact of grain boundaries on the vibrational properties of phononic crystals. 

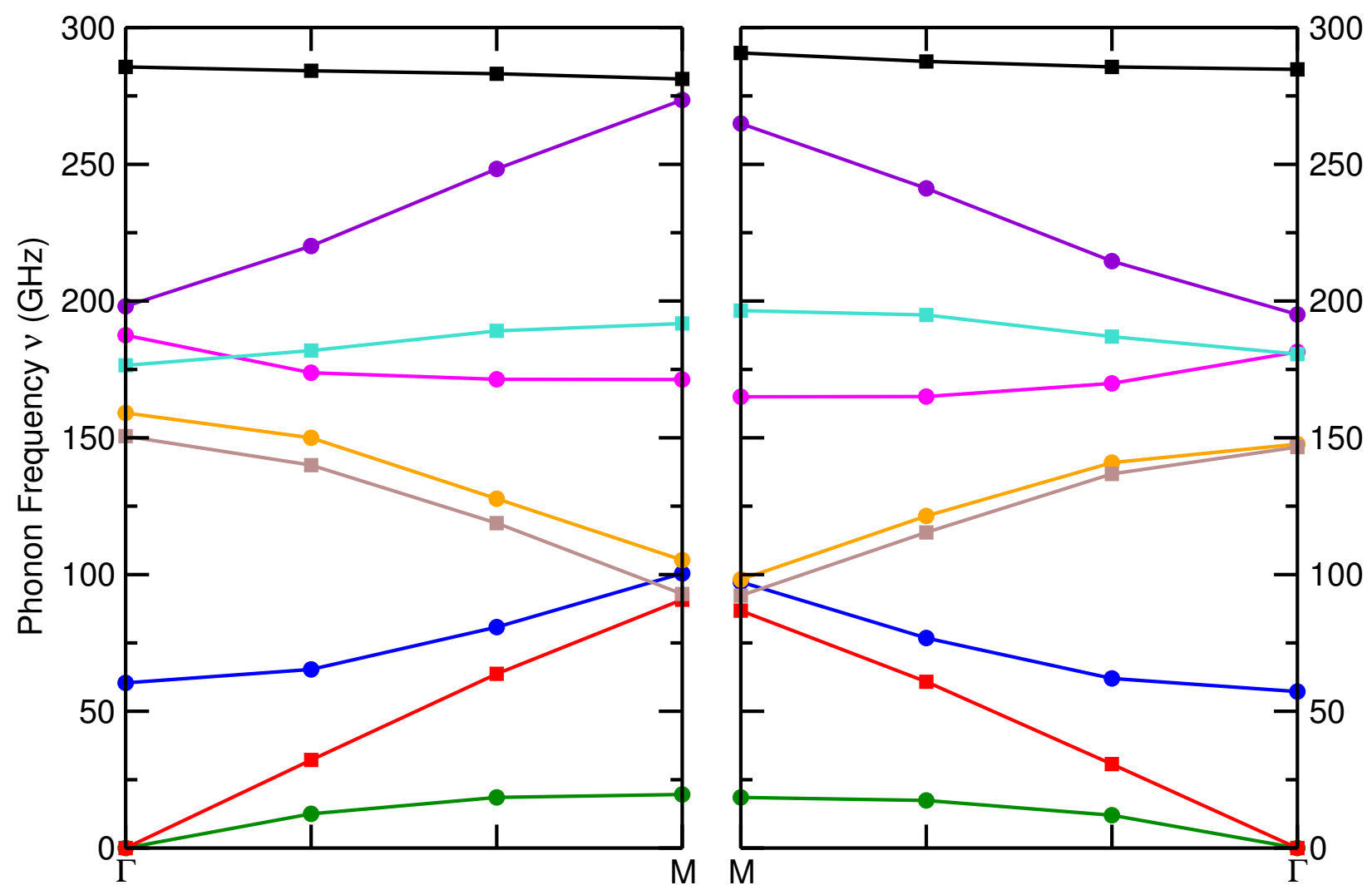

Figure 3. Vibrational band structure of single crystal (left panel) and polycrystalline (right panel) silicon phononic crystal models calculated from molecular dynamics simulations. Circles (squares) indicate modes derived from transversal (longitudinal) velocity data. Lines are only a guide to the eye.

The calculated vibrational densities of states show a softening effect of grain boundaries on the phononic crystal. In addition to this, the disorder present in the polycrystalline model reduces the number of sharp peaks in the density of states. Some peaks are, however, unaffected or even strengthened by the presence of grain boundaries which might be an indication that these peaks are caused by localized modes that are confined to the nanowires or nanoparticles.

The calculated vibrational band structures confirm the softening effect of the grain boundaries on the phononic crystals since most of the bands are shifted to lower frequencies in the polycrystalline phononic crystal. The effect of the grain boundaries on the bands is, however, not uniform and some modes are actually shifted to higher frequencies. A partial band gap is observed in both models along the $\Gamma-\mathrm{M}$ direction. This band is wider in the polycrystalline model.

Overall, the differences found in this study between the vibrational densities of states and the vibrational band structures of the two phononic crystals are not too big. This indicates that polycrystalline phononic crystals of the type studied here might be used successfully in signal processing applications. More work will be required to find out, to what extent grain boundaries affect the thermal transport through the systems. 


\section{ACKNOWLEDGMENTS}

Financial support by Laurentian University and the Natural Sciences and Engineering Research Council of Canada (NSERC) [grant number 371446-11] is gratefully acknowledged. This work has been made possible by generous allocation of computer time on the facilities of Compute/Calcul Canada.

\section{REFERENCES}

1. A. Khelif and A. Adibi (eds.), Phononic Crystals (Springer, Heidelberg, New York, 2016).

2. V. Laude, Phononic Crystals (De Gruyter, Berlin, Boston, 2015).

3. P.A. Deymier (ed.), Acoustic Metamaterials and Phononic Crystals (Springer, Berlin, Heidelberg, 2013).

4. J.N. Gillet, Y. Chalopin, and S. Volz, J. Heat Transfer 131, 043206 (2009).

5. J.K. Yu, S. Mitrovic, D. Tham, J. Varghese, and J.R. Heath, Nature Nanotech. 5, 718 (2010).

6. P. Hopkins, C.M. Reinke, M.F. Su, R.H. Olsson III, E.A. Shaner, Z.C. Leseman, J.R. Serrano, L.M. Phinney, and I. El-Kady, Nano Lett. 11, 107 (2011).

7. R. Meyer, Phys. Stat. Sol. A, doi: 10.1002/pssa.201600387 (2016).

8. M. I. Baskes, Phys. Rev. B 46, 2727 (1992).

9. W.C. Swope, H.C. Andersen, P.H. Berens, and K. R. Wilson, J. Chem Phys. 76, 637 (1982).

10. C.Z. Wang, A. Fasolino, and E. Tosatti, Phys. Rev. B 37, 2116 (1988). 